ORIGINAL ARTICLE

\title{
Growth hormone treatment in children with rheumatic disease, corticosteroid induced growth retardation, and osteopenia
}

\author{
F K Grote, L W A Van Suijlekom-Smit, D Mul, W C J Hop, R Ten Cate, W Oostdijk, \\ W Van Luijk, C J A Jansen-van Wijngaarden, S M P F De Muinck Keizer-Schrama
}

See end of article for authors' affiliations

Correspondence to: Mrs F K Grote, Dept of Pediatrics J6-S, Leiden University Medical Center, PO Box 9600, 2300 RC Leiden, Netherlands; f.k.grote@lumc.nl

Accepted

28 September 2005

Published Online First 13 October 2005

Background: In children with severe rheumatic disease (RD), treatment with corticosteroids (CS) is frequently needed and growth retardation and osteopenia may develop. A beneficial effect of human growth hormone $(\mathrm{hGH})$ has been reported but mostly in trials without a control group.

Aims: To study the effect of hGH on growth, bone mineral density (BMD), and body composition, taking the disease activity and CS use into account.

Methods: Randomised controlled trial on 17 prepubertal RD patients with growth retardation and/or decreased BMD. The $h G H$ group $(n=10)$ received treatment with $h G H 4 \mathrm{IU} / \mathrm{m}^{2} /$ day $(\sim 0.045 \mathrm{mg} / \mathrm{kg} /$ day) during two years. The controls $(n=7)$ received no $\mathrm{GH}$ treatment.

Results: During the two year study period the disease activity, and use of CS and methotrexate (MTX) did not differ between the groups. There was a significant mean increase in height standard deviation score (HSDS) in the $h G H$ group $(0.42 \pm 0.16 \mathrm{SDS})$ and a non-significant decrease in the controls $(-0.18 \pm 0.11 \mathrm{SDS})$. Change in BMD did not differ significantly between the groups, although the increase in BMD for lumbar spine within the $\mathrm{hGH}$ group was significant. Lean body mass improved significantly in the $\mathrm{hGH}$ group compared to controls $(0.64 \pm 0.19$ SDS versus $-0.20 \pm 0.17 \mathrm{SDS})$, while the decrease in percentage fat was not significant.

Conclusions: There was a significant effect of $\mathrm{hGH}$ on growth and lean body mass, but a longer duration of treatment might be necessary to evaluate the effect of $h G H$ on BMD.

R heumatic disease (RD) in childhood is a collective term for several chronic diseases that have an inflammatory origin and are usually associated with arthritis. Initial treatment consists of non-steroidal anti-inflammatory drugs in combination with corticosteroids (CS), sulfasalazine, methotrexate (MTX), and recently TNF $\alpha$ blocking agents ("biologicals", such as etanercept).

Of these medications, only MTX and prednisone have an effect on growth and bone mineral density. ${ }^{1}$ Negative side effects of long term daily administration of CS are a decline of growth velocity and osteoporosis. ${ }^{2}$ Diminished physical activity associated with arthritis negatively influences weight bearing and movement, both of which play a role in bone turnover. ${ }^{3}$ Moreover, chronic inflammation inhibits the GH-IGF-I, axis resulting in a decrease in bone mineral density (BMD) and growth retardation. ${ }^{4-6}$ In spite of the development of new treatments and efforts to avoid long term therapy with high doses of CS, their use is still inevitable in a subset of children with severe forms of RD.

Several authors have already reported the effect of human growth hormone on growth and BMD in children with rheumatic disease, treated with CS. $^{7-14}$ The reported studies included variable numbers of children and treatment periods. Most of the studies were uncontrolled trials and addressed either growth retardation, or BMD and body composition. The aim of this project was to study the effect of human growth hormone (hGH) on growth, BMD, and body composition in children with $\mathrm{RD}$, taking into account the disease activity and dosage of CS in a prospective randomised controlled trial.

\section{METHODS}

Patients

Between March 1998 and December 1999, prepubertal patients with RD and a decrease in height of more than 0.5
SDS since diagnosis and/or a BMD-SDS of the lumbar spine of $<-1.5$ SDS, and who were being treated with CS, were enrolled in a randomised controlled trial. Diagnostic criteria to be met were: the DURBAN criteria ${ }^{15}$ for juvenile idiopathic arthritis (JIA); the revised ACR criteria ${ }^{16}$ for systemic lupus erythematosus (SLE); the criteria of Sharp and colleagues ${ }^{17}$ for mixed connective tissue disease (MCTD); and the criteria of Bohan and Peter ${ }^{18}{ }^{19}$ for dermatomyositis. Exclusion criteria were associated diseases that might affect growth, interfere with therapy, or include CNS involvement. Written informed consent from the parents and approval by the local ethical committees were obtained.

\section{Treatment regime}

Patients were randomised to an hGH group receiving treatment with hGH $4 \mathrm{IU} / \mathrm{m}^{2} /$ day $(\sim 0.045 \mathrm{mg} / \mathrm{kg} /$ day $)$ during two years or a control group, receiving no GH treatment. The patients were stratified for age and height standard deviation score (HSDS). The study was performed in Erasmus MCSophia Children's Hospital; treatment of the RD was monitored by the patients' own paediatric rheumatologists in different hospitals. There were no restrictions in the prescribed medication and changes in treatment were justified. Participation in other studies was not allowed.

\section{Anthropometrical parameters}

Height was measured at three month intervals. Auxological data and data on CS and MTX use previous to the study were

\footnotetext{
Abbreviations: $B M D$, bone mineral density; $C S$, corticosteroid; $E S R$, erythrocyte sedimentation rate; $\mathrm{hGH}$, human growth hormone; HSDS, height standard deviation score; JIA, juvenile idiopathic arthritis; MCTD mixed connective tissue disease; MTX, methotrexate; RD, rheumatic disease; SDS, standard deviation score; SLE, systemic lupus erythematosus; VAS, visual analogue scale
} 
Table 1 Clinical characteristics of the patients at the start of the study

\begin{tabular}{lll}
\hline & GH treated group & Controls \\
\hline Sex (M:F) & $2: 8$ & $2: 5$ \\
Age at start (year) & $8.0(5.7$ to 13.0$)$ & $8.1(6.8$ to 10.8$)$ \\
Height SDS & $-1.4(-3.0$ to 0.1$)$ & $-1.9(-2.9$ to -1.0$)$ \\
BMD total body SDS & $-0.8(-1.8$ to 0.8$)$ & $-1.9(-2.5$ to -0.3$)$ \\
BMD lumbar spine SDS & $-1.6(-2.3$ to 0.6$)$ & $-1.9(-2.7$ to -0.5$)$ \\
Lean body mass SDS & $-1.9(-2.9$ to -0.0$)$ & $-1.9(-3.0$ to -0.9$)$ \\
\% fat SDS & $1.5(-0.6$ to 3.2$)$ & $0.0(-1.5$ to -3.9$)$ \\
Target height SDS & $0.4(-1.5$ to 3.1$)$ & $0.3(-1.8$ to 3.3$)$ \\
Cumulative dose of prednisone (mg) & $5963(2094-27864)$ & $7603(2317-26810)$ \\
Cumulative dose of methotrexate (MTX) (mg) & $814(180-2999)$ & $765(0-2184)$ \\
Physician global assessment of disease activity (mm) ${ }^{*}$ & $66(10-95)$ & $19.0(5-93)$ \\
Parent/patient assessment of the overall wellbeing (mm) & $26(0-49)$ & $19.0(0-45)$ \\
Functional ability (range: $0-3)$ & $0.9(0-2.8)$ & $1.8(0.1-3.0)$ \\
Number of joints with active arthritis (range: 0-75) & $9(1-30)$ & $2(0-18)$ \\
Number of joints with limited range of motion (range: 0-57) & $6(2-21)$ & $3(0-23)$ \\
ESR (mm/h) & $26.5(3-77)$ & $11.0(6-30)$ \\
IGF-I SDS & $0.3(-1.4$ to 0.8$)$ & $0.1(-0.9$ to 1.2$)$ \\
\hline Results expressed as median (range) & & \\
${ }^{*}$ Measured on a 100 mm visual analogue scale (VAS). & & \\
TChild Health Assessment Questionnaire (CHAQ). & & \\
\hline
\end{tabular}

collected retrospectively and expressed as SD scores and cumulative doses. Height, target height, and body mass index (BMI) were expressed as SDS, using the Dutch reference growth data. ${ }^{21}$ Puberty was assessed using the stages of Tanner and Whitehouse.22 Bone age (BA) was calculated every six months using a segmented Greulich and Pyle (GP) score. ${ }^{23}$

\section{Parameters of bone mineral density and body composition}

The BMD $\left(\mathrm{g} / \mathrm{cm}^{2}\right)$ of lumbar spine and total body and body composition were measured every six months by dual energy x-ray absorptiometry (DEXA) (Lunar, DPXL/PED). Results were compared with age and sex matched reference values. ${ }^{24}$ $\mathrm{BMD}$ of lumbar spine was corrected for bone size (BMAD). ${ }^{25}$ Additionally all BMD and body composition parameters were corrected for bone age.

\section{Parameters of disease activity}

Disease activity was measured six monthly with the separate variables of the PRINTO (Pediatric Rheumatology Trial Organisation) core set, ${ }^{26}$ including the following six endpoints: (1) physician global assessment of disease activity (measured on a $100 \mathrm{~mm}$ visual analogue scale (VAS)); (2) parent/patient assessment of the overall wellbeing (VAS); (3) functional ability (measured by Child Health Assessment Questionnaire (CHAQ), ascending range $\left.0-3^{27}\right)$; (4) number of joints with active arthritis (range: $0-75) ;(5)$ number of joints with limited range of motion (range: 0-57); (6) erythrocyte sedimentation rate (ESR) $(\mathrm{mm} / \mathrm{h})$.

The evaluation was done by an experienced paediatric physiotherapist except for the physician global assessment, which was carried out by the patient's own paediatric rheumatologist. In spite of the fact that some patients with SLE and MCTD have no joint involvement, we decided to use the variables of the PRINTO score for all our patients for comparability.

\section{Laboratory parameters}

Before the start of the study, a GH stimulation test with arginine was performed. Serum parameters of growth and bone metabolism measured six monthly were: PTH, 1,25-dihydroxyvitamin D, 25-hydroxyvitamin D, carboxy terminal telopeptide of type I collagen (ICTP), procollagen type I C-terminal propeptide (PICP), calcium, alkaline phosphatase, inorganic phosphate, creatinine, IGF-I, and IGFBP3. ${ }^{28}$ These parameters were expressed as sex and age matched SDS using our own reference values. ${ }^{29}$ Additionally FT4 and TSH were measured.
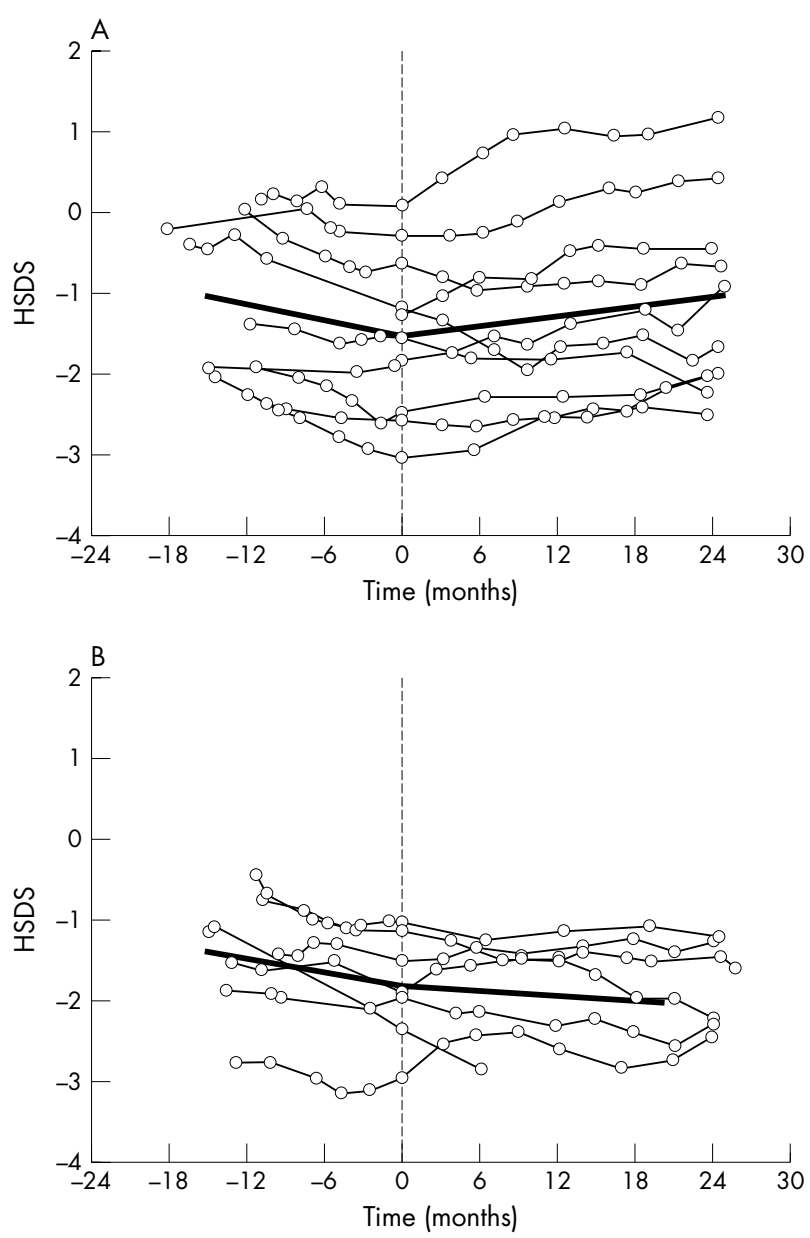

Figure 1 Individual levels of height SDS per group before and during the study period. (A) $\mathrm{GH}$ treated group. (B) Controls. Time $=0$ denotes the start of the study. The solid line represents the regression line of HSDS within the group with a breakpoint at time $=0$.

\section{Safety parameters}

Oral glucose tolerance tests (OGTT) were performed at the start and end of the study. The following definition of impaired glucose tolerance was used: the 120 minute level was $>7.8 \mathrm{mmol} / \mathrm{l}(140 \mathrm{mg} / \mathrm{dl})$ and $<11.1 \mathrm{mmol} / \mathrm{l}(200 \mathrm{mg} / \mathrm{dl}){ }^{30}$ 
Table 2 Mean annual change $( \pm S E M)$ in bone mineral density and body composition during two years of study

\begin{tabular}{lccc}
\hline Parameter & GH treated group & Controls & $\begin{array}{c}\text { GH treated } v \text { controls } \\
\text { (p value) }\end{array}$ \\
\hline BMD SDS for total body & $-0.09 \pm 0.07$ & $-0.14 \pm 0.14$ & 0.58 \\
BMD SDS for lumbar spine & $0.26 \pm 0.11^{*}$ & $0.12 \pm 0.13$ & 0.31 \\
BMAD SDS for lumbar spine & $0.07 \pm 0.13$ & $-0.22 \pm 0.20$ & 0.22 \\
Lean body mass & $0.32 \pm 0.10^{* *}$ & $-0.10 \pm 0.09$ & $<0.01$ \\
$\%$ fat & $-0.26 \pm 0.13$ & $-0.14 \pm 0.36$ & 0.76 \\
BMI SDS & $-0.08 \pm 0.17$ & $0.12 \pm 0.21$ & 0.46 \\
\hline Within group significance: ${ }^{*} \mathrm{p}<0.05,{ }^{* *} \mathrm{p}<0.01$ & &
\end{tabular}

\section{Statistical analysis}

Random coefficient models (SAS PROC MIXED) were used to evaluate the effect of hGH on change in HSDS, bone mineral density, and body composition. For HSDS, peacewise linear regression was also done using pre-trial data. ${ }^{31}$ Repeated measurement ANOVA was used to evaluate the effect on the laboratory parameters. The Mann-Whitney U test was used to evaluate the difference between groups in mean daily doses (mg/day) of CS and MTX and the parameters of the PRINTO core set during the study. Spearman's correlation coefficients were used to test the relation between the change from baseline of HSDS after two years and several parameters (prednisone dosage, VAS, GH peak levels after stimulation, ESR, and the changes from baseline of IGF-I SDS, BMD SDS for lumbar spine, and ALP SDS after two years). Similar coefficients were used to test the relation between the change from baseline of BMD SDS for the lumbar spine and the change from baseline of ALP SDS after two years. Values of $p$ (two sided) which were $\leqslant 0.05$ were considered statistically significant. Power calculations had led to 20 patients. However, due to a lower accrual than expected, we decided to stop the inclusion after 17 patients. This decision was taken before all data were gathered and analysed. With this reduced number, differences regarding the change from baseline of HSDS between groups, expressed as effect size (that is, difference of means divided by the standard deviation), can be detected with power $80 \%$ if the effect size equals 1.4 .

\section{RESULTS}

\section{Patients}

The ratio hGH treated patients to controls after randomisation was 10:7. There were nine children with systemic JIA (hGH group:controls $=7: 2$ ), two with polyarticular JIA ( $1: 1$ ), two with MCTD (1:1), two with SLE (1:1), and two with dermatomyositis $(0: 2)$. Sixteen patients completed the study. Although one patient (control group) discontinued the study after six months because of bone marrow transplantation, the data acquired were included in the analyses. During the study five children reached puberty (three from the hGH group). The clinical characteristics of the patients at the start of the study are shown in table 1. The two groups were comparable at baseline.

Three children had a decreased GH peak (<20 IU/l) after stimulation, with normal IGF-I and IGFBP3 serum levels (hGH group:controls $=1: 2$ ).

\section{Growth and bone age}

A significant mean increase of HSDS was seen in the hGH group of $0.42 \pm 0.16$ SDS $(p=0.03)$ and a non-significant decrease in the controls of $-0.18 \pm 0.11$ SDS, resulting in a difference of $0.6 \pm 0.19$ SDS between the groups $(p=0.02)$.

When pre-trial growth data were included in the analysis, there was a significant change in growth at the start of the trial within the hGH group and no significant change within the control group (fig 1 ).

With correction for bone age the difference in increase in HSDS between the groups becomes even more significant $(p=0.004)$. The ratio change in bone age to change in chronological age was not significantly different from one in both groups.

\section{Bone mineral density and body composition}

The hGH group showed a significant mean increase of the BMD SDS for the lumbar spine of $0.52 \pm 0.22$ SDS during two years. This significance was no longer perceptible after correction for bone size. BMD for total body did not increase significantly. Controls showed no significant changes in BMD parameters. Finally, changes in BMD between the groups were not significantly different (table 2 ).

Lean body mass increased significantly in the hGH group compared to controls $(0.64 \pm 0.19$ SDS versus $-0.20 \pm 0.17$ SDS in two years, $\mathrm{p}<0.01$ ) (table 2). Neither the decrease in percentage fat nor the change in BMI SDS was significantly different between groups. Results were similar after correction for bone age.

Table 3 Values of the different variables of the PRINTO core set and medication use per day during two years of study

\begin{tabular}{|c|c|c|c|}
\hline Parameter & GH treated group & Controls & $\begin{array}{l}\text { GH treated } \\
v \text { controls } \\
\text { (p value) }\end{array}$ \\
\hline Physician global assessment of disease activity (VAS) & $41(18.8-61.6)$ & $18(10.2-66.4)$ & 0.23 \\
\hline Parent/patient assessment of overall wellbeing (VAS) & $10.2(0.8-36)$ & $15.7(1-37.6)$ & 0.75 \\
\hline Functional ability & $1.4(0.1-2.3)$ & $1.7(0.3-2.7)$ & 0.19 \\
\hline Number of joints with active arthritis & $4.4(0.4-20.0)$ & $2.6(0-22.4)$ & 0.33 \\
\hline Number of joints with limited range of motion & $7.2(2.8-16.4)$ & $6.6(2.2-23.2)$ & 0.96 \\
\hline ESR & $24.1(8.5-70.9)$ & $12.4(11.3-23)$ & 0.08 \\
\hline Prednisone (mg/day) & $6.1(0-29.2)$ & $8.0(0.1-24)$ & 0.52 \\
\hline MTX (mg/day) & $2.4(0.7-2.9)$ & $2.3(0-5.3)$ & 0.64 \\
\hline
\end{tabular}

Results expressed as medians (range of individual mean values during the study). 


\section{Medication, disease activity, and laboratory parameters}

There was no significant difference in MTX, prednisone dose, or the different variables of disease activity between the groups (table 3 ).

The mean difference in alkaline phosphatase, IGF-I, and IGFBP3 SDS were significant between the groups. The other biochemical markers of growth and bone metabolism were not significantly different (see table 4 ).

\section{Correlations}

There was a significant negative correlation $(\mathrm{r}=-0.61$; $\mathrm{p}=0.012$ ) between the dosage of prednisone and the change from baseline of HSDS after two years in the combined groups (hGH treated and controls). This relation did not differ significantly between the groups. No other significant correlations were found.

\section{Safety parameters}

Treatment was well tolerated and no drug related adverse events were seen during the study period. Nine children (five in the hGH group) showed impaired glucose tolerance at start of the study. Only three had impaired glucose tolerance after two years and no new cases were observed. None of the children developed diabetes mellitus.

\section{DISCUSSION}

Our study is the first trial with a control group where the effect of growth hormone on growth as well as on BMD and body composition is studied. A significant positive effect of growth hormone on height SDS was seen, irrespective of disease activity, dosage of steroids and of MTX, without undue acceleration of bone maturation.

Although there was a significant increase in BMD for the lumbar spine in the hGH group, there was no significant effect of growth hormone detectable on BMD compared with controls. On the other hand we found a significant effect on body composition, especially on lean body mass. Since the most important factor in the activation of the skeletal system is the strain of bones due to muscle contraction, ${ }^{32}$ an increase of lean body mass might positively influence bone mineral density in the long run.

To correct for other factors influencing growth retardation and osteopenia we studied the disease itself and other medications such as CS and MTX. In our study there was a significant negative correlation between prednisone and the change from baseline of HSDS after two years. Since there was no significant correlation between ESR and change from baseline of HSDS after two years in the whole group or a significant difference between the groups, the significant

\begin{tabular}{|c|c|c|}
\hline Parameter & $\begin{array}{l}\text { Difference of } \\
\text { means (GH treated } \\
\text { minus controlss) }\end{array}$ & $\begin{array}{l}\text { GH treated } v \\
\text { controls (p } \\
\text { value) }\end{array}$ \\
\hline ICTP SDS & $1.2 \pm 0.6$ & 0.06 \\
\hline PICP SDS & $0.9 \pm 0.5$ & 0.06 \\
\hline Alkaline phosphatase SDS & $1.0 \pm 0.4$ & 0.03 \\
\hline Phosphate SDS & $0.6 \pm 0.4$ & 0.19 \\
\hline Ca SDS & $0.2 \pm 0.4$ & 0.59 \\
\hline 1,25-dihydroxyvitamin $D_{3}$ SDS & $0.5 \pm 0.2$ & 0.05 \\
\hline 25 -hydroxyvitamin $D_{3} S D S$ & $-0.4 \pm 0.3$ & 0.16 \\
\hline IGFBP3 SDS & $0.8 \pm 0.3$ & 0.01 \\
\hline IGF-I SDS & $1.1 \pm 0.3$ & 0.003 \\
\hline FT4 SDS & $-0.04 \pm 0.4$ & 0.9 \\
\hline TSH SDS & $0.1 \pm 0.7$ & 0.9 \\
\hline
\end{tabular}

increase in height SDS was most likely to be an effect of growth hormone. This was also illustrated by the nonsignificant difference in disease activity and the use of prednisone or MTX between the groups.

The significant improvement in height found in this study concurs with other publications. ${ }^{7-14}$ Most are case series, of 7-20 patients, with an increase of growth velocity varying between $1.9 \mathrm{~cm}$ and $6.7 \mathrm{~cm}$ per year during growth hormone treatment. These discordant results can partly be explained by the inclusion of pubertal patients and the different hGH dosages varying from 0.57 to $2 \mathrm{mg} / \mathrm{m}^{2} /$ day. Differences in the severity of the disease may also play a role. Bechtold et al conducted one of the two other known controlled trials to study the effect of growth hormone on growth retardation. ${ }^{8}$ They found a relative height gain of 1.7 SDS over four years. This is slightly more than the difference in increase in HSDS of $0.6 \pm 0.19$ SDS over two years we found in our study and also more than the 0.13 SDS over six months which Saha et al found in their placebo controlled crossover study. ${ }^{13}$ These differences in height gain might be due to the different duration of the study, difference in severity of the disease, different dosage of prednisone, or the difference in baseline HSDS. In the study of Bechtold et al, for example, the children were shorter at the start than in our study $(-3.3$ versus -1.9 in the control group and -2.3 versus -1.4 in the hGH group). This last hypothesis could however not be supported by our study in which no correlation was found between the baseline HSDS and the change from baseline of HSDS after two years.

The effect of hGH on bone mineral density, bone turnover, and body composition has already been described in the literature, but was hardly ever studied in a controlled trial. Although Rooney et al found an increase in bone mineral content (expressed per $\mathrm{cm}$ of vertebral height BMD, in $\mathrm{g} / \mathrm{cm}),{ }^{10}$ they did not evaluate the BMD $\left(\mathrm{g} / \mathrm{cm}^{2}\right)$ itself or $\mathrm{BMAD}$, which are area densities derived from the bone mineral content that correct even better for bone size. In contrast to a previous study, ${ }^{12}$ Czernichow et al found in their second, longer term study, an increase in bone mineral density during treatment. ${ }^{14}$ It is however questionable whether the increase in this study might have been age related, since the SD scores for bone mineral density were expressed for weight and not for age. Also there was no control group available to be able to attribute this increase to the effect of growth hormone. The fact that there is no significant effect of hGH on bone mineral density in our study, while a significant effect on lean body mass was present, may indicate that a longer duration of treatment is necessary to evaluate the effect on bone mineral density. This is also supported by Bechtold et al, who found despite an increase in bone turnover, only a stabilisation of bone mineral density. ${ }^{33}$

It is assumed that disturbances in the GH-IGF-I axis is one of many factors contributing to growth retardation in children with rheumatic disease. ${ }^{12}{ }^{34-37}$ However, in our study no obvious disturbances in this axis were noted. This implies that the significant effect of growth hormone on growth is not explained by this phenomenon.

This study is limited by the heterogeneity in the study population and the small number of patients. We managed however to show the effect of growth hormone on growth, bone density, and body composition by using age and sex matched references and the same core set variables for disease activity for the different diseases.

In conclusion, hGH has a significant effect on growth, irrespective of the disease activity and the dosage of steroids and MTX used. There is also a significant effect on body composition (especially on lean body mass), but a longer duration of treatment might be necessary to evaluate the effect of hGH on bone mineral density. 


\section{What is already known on this topic}

- In children with severe rheumatic disease, a beneficial effect of hGH on corticosteroid induced growth retardation has been reported, mostly in trials without a control group

- The effect of hGH on bone mineral density, bone turnover, and body composition has previously been described, but remains questionable

\section{ACKNOWLEDGEMENTS}

We acknowledge Ingrid van Slobbe, research nurse, for her great attribution to the study, Pfizer for financial support, and all children and their parents who participated in the study.

\section{Authors' affiliations \\ F K Grote, L W A Van Suijlekom-Smit, D Mul, S M P F De Muinck}

Keizer-Schrama, Dept of Paediatrics, Erasmus MC-Sophia Children's

Hospital, Rotterdam, Netherlands

R Ten Cate, W Oostdijk, Dept of Paediatrics, Leiden University Medical Center, Leiden, Netherlands

W Van Luijk, Dept of Paediatrics, University Hospital Groningen, Groningen, Netherlands

W C J Hop, Dept of Epidemiology \& Biostatistics, Erasmus MC, University Medical Centre Rotterdam, Rotterdam, Netherlands C J A Jansen-van Wijngaarden, Dept of Paediatric Physiotherapy, Erasmus MC-Sophia Children's Hospital, Rotterdam, Netherlands Competing interests: none declared

\section{REFERENCES}

1 Falcini $F$, Ermini $M$, Bagnoli $F$. Bone turnover is reduced in children with juvenile rheumatoid arthritis. J Endocrinol Invest 1998;21:31-6.

2 Mushtaq T, Ahmed SF. The impact of corticosteroids on growth and bone health. Arch Dis Child 2002;87:93-6.

3 Mul D, Suijlekom-Smit LWA, ten Cate R, et al. Bone mineral density and body composition and influencing factors in children with rheumatic diseases treated with corticosteroids. J Pediatr Endocrinol Metab 2002;15:187-92.

4 Davies UM, Rooney M, Preece MA, et al. Treatment of growth retardation in juvenile chronic arthritis with recombinant human growth hormone. Sheumatol 1994;21:153-8.

5 Hopp RJ, Degan J, Corley K, et al. Evaluation of growth hormone secretion in children with juvenile rheumatoid arthritis and short stature. Nebr Med J 1995;80(3):52-7

6 Woo PM. Growth retardation and osteoporosis in juvenile chronic arthritis. Clin Exp Rheumatol 1994;12(suppl 10):87-90.

7 Al Mutair A, Bahabri S, Al Mayouf S, et al. Efficacy of recombinant human growth hormone in children with juvenile rheumatoid arthritis and growth failure. J Pediatr Endocrinol Metab 2000;13:899-905.

8 Bechtold S, Ripperger P, Hafner R, et al. Growth hormone improves height in patients with juvenile idiopathic arthritis: 4-year data of a controlled study. J Pediatr 2003;143:512-19

9 Butenandt $O$. Rheumatoid arthritis and growth retardation in children: treatment with human growth hormone. Eur J Pediatr 1979;130:15-28.

10 Rooney M, Davies UM, Reeve J, et al. Bone mineral content and bone mineral metabolism: changes after growth hormone treatment in juvenile chronic arthritis. J Rheumatol 2000;27:1073-81.

11 Svantesson H. Treatment of growth failure with human growth hormone in patients with juvenile chronic arthritis. A pilot study. Clin Exp Rheumatol $1991 ; 9$ (suppl 6):47-50.

12 Touati G, Prieur AM, Ruiz JC, et al. Beneficial effects of one-year growth hormone administration to children with juvenile chronic arthritis on chronic steroid therapy. I. Effects on growth velocity and body composition. J Clin Endocrinol Metab 1998;83:403-9.

13 Saha MT, Haapasaari J, Hannula S, et al. Growth hormone is effective in the treatment of severe growth retardation in children with juvenile chronic arthritis. Double blind placebo-controlled followup study. J Rheumatol 2004;31:1413-17.

\section{What this study adds}

- This randomised controlled trial shows a significant effect of hGH on growth, irrespective of the disease activity and the dosage of steroids and MTX used

- There is a significant effect on body composition (especially on lean body mass), but a longer duration of treatment might be necessary to evaluate the effect of $\mathrm{hGH}$ on bone mineral density

14 Simon D, Lucidarme N, Prieur AM, et al. Effects on growth and body composition of growth hormone treatment in children with juvenile idiopathic arthritis requiring steroid therapy. J Rheumatol 2003;30:2492-9.

15 Petty RE, Southwood TR, Baum J, et al. Revision of the proposed classification criteria for juvenile idiopathic arthritis: Durban, 1997. J Rheumatol 1998;25:1991-4

16 Tan EM, Cohen AS, Fries JF, et al. The 1982 revised criteria for the classification of systemic lupus erythematosus. Arthritis Rheum 1982;25:1271-7.

17 Sharp GC, Irvin WS, Tan EM, et al. Mixed connective tissue disease-an apparently distinct rheumatic disease syndrome associated with a specific antibody to an extractable nuclear antigen (ENA). Am J Med 1972;52:148-59

18 Bohan A, Peter JB. Polymyositis and dermatomyositis (second of two parts). N Engl J Med 1975;292:403-7.

19 Bohan A, Peter JB. Polymyositis and dermatomyositis (first of two parts). N Engl J Med 1975;292:344-7.

20 Fredriks AM, van Buuren S, Burgmeijer RJ, et al. Continuing positive secular growth change in the Netherlands 1955-1997. Pediatr Res 2000;47:316-23.

21 Fredriks AM, van Buuren S, Wit JM, et al. Body index measurements in 1996-7 compared with 1980. Arch Dis Child 2000;82:107-12

22 Tanner JM, Whitehouse RH. Clinical longitudinal standards for height, weight, height velocity, weight velocity, and stages of puberty. Arch Dis Child 1976;51:170-9.

23 Mul D, Oostdijk W, Waelkens JJ, et al. Gonadotrophin releasing hormone agonist treatment with or without recombinant human $\mathrm{GH}$ in adopted children with early puberty. Clin Endocrinol (Oxf) 2001;55:121-9.

24 van der Sluis I, de Ridder MA, Boot AM, et al. Reference data for bone density and body composition measured with dual energy $\mathrm{x}$ ray absorptiometry in white children and young adults. Arch Dis Child 2002;87:341-7.

25 Kroger H, Vainio P, Nieminen J, et al. Comparison of different models for interpreting bone mineral density measurements using DXA and MRI technology. Bone 1995;17:157-9.

26 Giannini EH, Ruperto N, Ravelli A, et al. Preliminary definition of improvement in juvenile arthritis. Arthritis Rheum 1997;40:1202-9

27 Singh G, Athreya BH, Fries JF, et al. Measurement of health status in children with juvenile rheumatoid arthritis. Arthritis Rheum 1994;37:1761-9.

28 van der Sluis I, Boot AM, Nauta J, et al. Bone density and body composition in chronic renal failure: effects of growth hormone treatment. Pediatr Nephrol 2000;15:221-8.

29 van der Sluis I, Hop WC, van Leeuwen JP, et al. A cross-sectional study on biochemical parameters of bone turnover and vitamin $d$ metabolites in healthy Dutch children and young adults. Horm Res 2002;57:170-9.

30 Report of the Expert Committee on the Diagnosis and Classification of Diabetes Mellitus. Diabetes Care 1997;20:1183-97.

31 Neter J, Wasserman W. Peacewise regression. Applied statistical models. Homewood, IL: Irwin RD, 1974:313-16.

32 Schonau E, Werhahn E, Schiedermaier U, et al. Influence of muscle strength on bone strength during childhood and adolescence. Horm Res 1996:45(suppl 1):63-6.

33 Bechtold S, Ripperger P, Bonfig W, et al. Bone mass development and bone metabolism in juvenile idiopathic arthritis: treatment with growth hormone for 4 years. J Rheumatol 2004;31:1407-12.

34 Aitman TJ, Palmer RG, Loftus J, et al. Serum IGF-I levels and growth failure in juvenile chronic arthritis. Clin Exp Rheumatol 1989:7:557-61.

35 Bennett AE, Silverman ED, Miller JJ III, et al. Insulin-like growth factors I and II in children with systemic onset juvenile arthritis. J Rheumatol 1988;15:655-8.

36 Davies UM, Jones J, Reeve J, et al. Juvenile rheumatoid arthritis. Effects of disease activity and recombinant human growth hormone on insulin-like growth factor 1, insulin-like growth factor binding proteins 1 and 3, and osteocalcin. Arthritis Rheum 1997;40:332-40.

37 Underwood LE. Growth retardation in chronic diseases: possible mechanisms. Acta Paediatr Suppl 1999;88(428):93-6. 